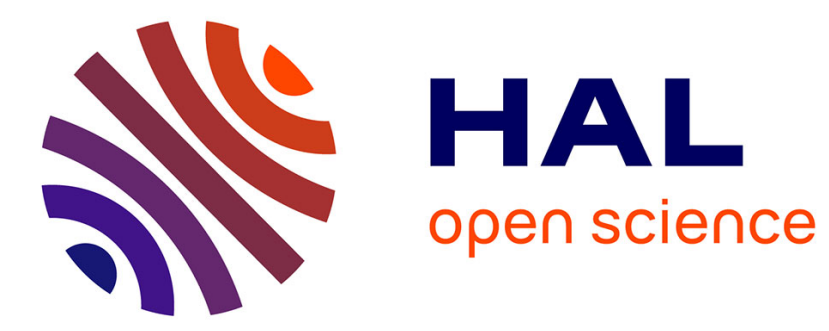

\title{
Methodology of optimisation for a nanostructured two-photon absorption photodetector
}

\author{
Maxence Dauphin, Baptiste Fix, Julien Jaeck, Riad Haïdar
}

\section{To cite this version:}

Maxence Dauphin, Baptiste Fix, Julien Jaeck, Riad Haïdar. Methodology of optimisation for a nanostructured two-photon absorption photodetector. Journal of the European Optical Society: Rapid publications, 2021, 17, pp.20. 10.21203/rs.3.rs-492917/v1 . hal-03282718v2

\section{HAL Id: hal-03282718 \\ https://hal.science/hal-03282718v2}

Submitted on 24 Jan 2022

HAL is a multi-disciplinary open access archive for the deposit and dissemination of scientific research documents, whether they are published or not. The documents may come from teaching and research institutions in France or abroad, or from public or private research centers.
L'archive ouverte pluridisciplinaire HAL, est destinée au dépôt et à la diffusion de documents scientifiques de niveau recherche, publiés ou non, émanant des établissements d'enseignement et de recherche français ou étrangers, des laboratoires publics ou privés. 


\title{
Methodology of optimisation for a nanostructured two-photon absorption photodetector
}

\author{
Maxence Dauphin ${ }^{1}$, Baptiste Fix ${ }^{1 *}$ (D) Julien Jaeck ${ }^{1}$ and Riad Haïdar ${ }^{1,2}$
}

\begin{abstract}
We introduce a 3-step method to optimise a nanostructured photodetector for infrared sensing through non degenerated two-photon absorption (NDTPA). First, the nanostructure is designed to tailor the distribution and concentration of both pump and signal intensities within the absorbing layer, thus leading to a gain in two-photon absorption. Second, the issue of the competition between NDTPA and other sub-bandgap transitions is tackled with a new figure of merit to favor as much as possible NDTPA while minimising other absorption processes. Third, a refined computation of the gain and the figure of merit is done to consider focused beams. Finally, two scenarios based on low power infrared photodetection are investigated to illustrate the flexibility and adaptibility of the method. It is shown that the gain is up to 7 times higher and the figure of merit is up to 20 times higher compared to the literature.
\end{abstract}

Keywords: Two-photon absorption, Nanostructures, Infrared photodetection

\section{Introduction}

Quantum infrared photodetection is classically done through direct absorption of a photon to enable an electronic transition in so-called linear light sensors. Several photodetection technologies are currently in competition in the mid-wave (3-5 $\mu \mathrm{m})$ and long-wave (7-12 $\mu \mathrm{m})$ infrared spectrum such as the bulk semiconductor technologies $\mathrm{HgCdTe}$ [1] and InSb [2], the quantum-well structures (QWIP) [3-5], or the type II super-lattices (T2SL) [6, 7]. Yet, owing to the small energy of infrared photons and thus of the electronic transition, background limited performances (BLIP) are only achieved at cryogenic temperature. The needed cooling machine limits the operability and the mean time before failure of the infrared photodetectors.

In parallel to the race toward higher operating temperature (HOT) of linear light sensors [8], new photodetection paradigm using non-linearities have been proposed either at the second [9] or third order processes [10-12]. Among

*Correspondence: baptiste.fix@onera.fr

'DOTA, ONERA, Université Paris-Saclay, 91123 Palaiseau, France

Full list of author information is available at the end of the article these third order phenomenons, two-photon absorption in higher band-gap energy semiconductors presents interesting properties for room temperature infrared photodetection. It consists in the quasi-simultaneous absorption of two photons [13] which makes it naturally interesting for high frequency and heterodyne application $[10,11]$. However, the low quantum efficiency of the mechanism [14] remains a strong limitation.

In order to tackle this issue, the nanostructures have been used for photon-harvesting [15], photon-trapping (MIM [16], GMR [17], etc.) and to locally enhance the electrical field [18]. In the case of infrared photodetection, these properties have proven to enhance sensing performances [19-21], mainly thanks to the reduction of the absorbing semiconductor volume. As well, the low efficiency of two-photon absorption can be improved thanks to the concentration ability of nanostructures [12] - even in the challenging case of bi-spectral optimisation. Indeed, an adapted nanostructuration makes it possible to concentrate and colocalise each beam intensities, leading to a gain and consequently to greater photogenerated currents [22]. 


\section{Discussion}

In this paper, we describe our methodology to optimise two-photon infrared photodetection in nanostructured detectors. First, we explain and quantify how nanostructures can enhance two-photon absorption (especially non-degenerated two-photon absorption called NDTPA) through fields concentration and colocalisation. Then, the competition between NDTPA and other sub-bandgap transitions is discussed, leading to the definition of a figure of merit for the design of the diodes. This figure of merit is then refined to tackle the issue of focused optical beams. Finally, the figure of merit is adapted to address a selection of low power infrared applications, namely monochromatic photodetection and infrared imaging.

Two photon infrared photodetection has been previously discussed in the literature [12, 22-24] with reasonable success. Yet, up to now the low responsivity and signal-to-noise ratio (SNR) have limited this technology to either short-pulsed laser sources or elaborated lab experiments. Based on one of these most recent works [22], we will revisit the optimisation process of a nanostructured detector design in order to take full benefit of the nanostructures for low power infrared photodetection. We will focus on the absorption process ; the transport and carrier collection will not be covered. The concepts discussed here will thus be illustrated on the photodiode characterised in [22], numerically simulated by the linear B-Spline modal method [25]. As illustrated in Fig. 1, the photodetector is a PIN diode made of Indium Phosphide (InP). Since the structure is supposed infinite along the $y-$ direction and infinitely periodic along the $\mathrm{x}$-direction, the computation can be reduced to the PIN area $L=d * h$. $\mathrm{InP}$ is known to be transparent in the infrared spectrum (bandgap energy $E_{g}=1.344 \mathrm{eV}[26,27]$ ) and to exhibit a high third order non-linear susceptibility [28]. For the simplicity and generalisation of the discussion, the PIN junction will be considered as a homogeneous absorbing medium. The InP allows to absorb simultaneously a 3.39 $\mu \mathrm{m}$ signal photon $\left(\hbar \omega_{s}=0.36 \mathrm{eV}\right)$ with a $1.06 \mu \mathrm{m}$ pump photon $\left(\hbar \omega_{p}=1.17 \mathrm{eV}\right)$ since $\hbar \omega_{s}+\hbar \omega_{p} \geq E_{g}$. This leads to a local carrier generation within the material, which will be swept out by the applied electric field and may participate to the two-photon photocurrent, which writes as :

$$
j_{\mathrm{NDTPA}} \sim \beta\left(\omega_{p}, \omega_{s}\right) i_{p} i_{s}
$$

with $\beta$ the two-photon absorption coefficient [13], $i_{p}$ the local pump intensity, and $i_{s}$ the local signal intensity.

Nanostructured electrodes are used to tailor both the concentration and colocalisation of the two intensities inside the diode. The concentration of optical fields is defined as a locally intense area of light. The colocalisation of optical fields is defined as the overlap at the same location of several fields of different wavelengths. Then, under the pump power non depletion hypothesis, the total current generated through NDTPA in the surface of the absorbing layer is :

$$
J_{\mathrm{NDTPA}}=\int_{\mathrm{L}} j_{\mathrm{NDTPA}} d S \sim G \beta\left(\omega_{p}, \omega_{s}\right) P_{p}^{(i)} P_{s}^{(i)}
$$

with $P_{p}^{(i)}$ and $P_{s}^{(i)}$ incident pump and signal linear power over one period, $L$ the area of the absorbing region.

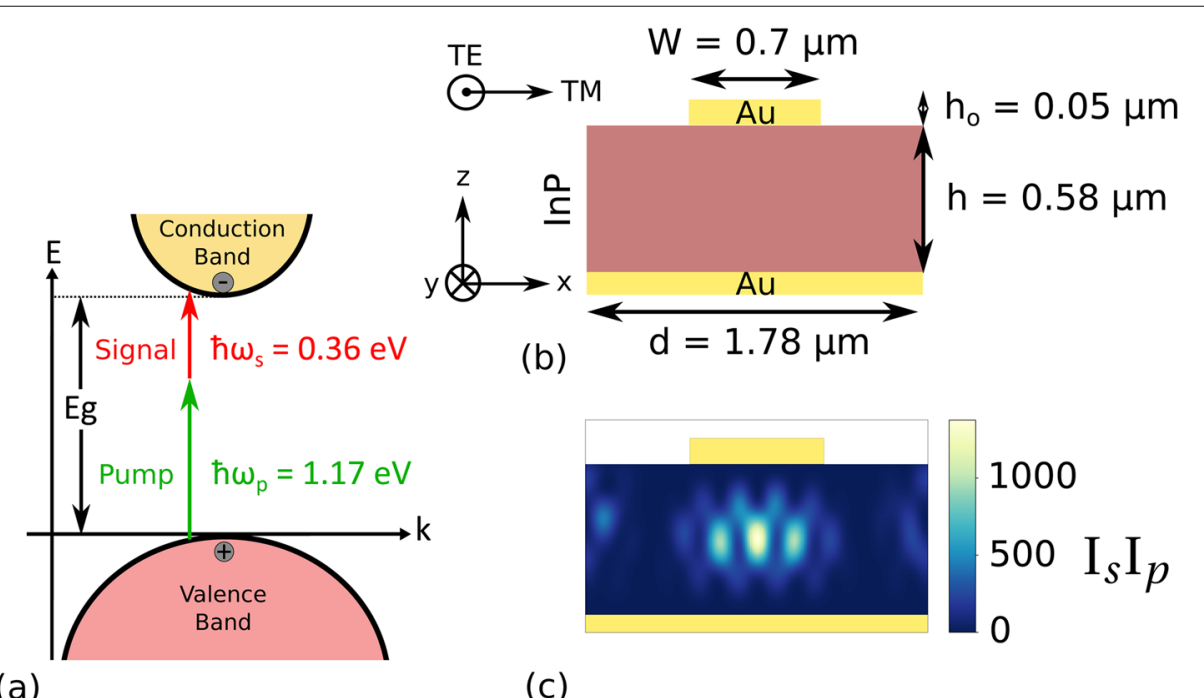

Fig. 1 (a). Scheme of NDTPA in a direct bandgap semiconductor. (b). Drawing of a published nanostructured photodiode designed for infrared photodetection [22] (c). Intensity map of signal and pump colocalisation 
Finally, $G$ is the dimensionless nanostructure gain which is formulated as :

$$
\begin{aligned}
& G=\int_{L} \frac{i_{p}(x, y) i_{s}(x, y)}{P_{p}^{(i)} P_{s}^{(i)}} d S=\int_{L} I_{p} I_{s} d S \text { with } I_{p}=\frac{i_{p}}{P_{p}^{(\mathrm{i})}} \\
& \quad \text { and } I_{s}=\frac{i_{s}}{P_{s}^{(\mathrm{i})}}
\end{aligned}
$$

The intensity map on Fig. 1.c illustrates the capability of the nanostructure to concentrate and colocalise the intensities $I_{p}$ and $I_{s}$, thus increasing the TPA by a gain $G=$ 120 for the optimal optical configuration (a collimated TE polarised $3.39 \mu \mathrm{m}$ signal at normal incidence and a collimated TE-polarised pump at $1.06 \mu \mathrm{m}$ pump with an angle of incidence of $11.83^{\circ}$ ). However, other pump related subbandgap processes have been shown to compete with NDTPA [29-31], leading to a higher background current and thus a lower SNR especially for low signal intensity.

The total carrier generation given in Eq. 4 is made of three contributions. The first one comes from the NDTPA. The second term is the degenerated two-photon absorption (DTPA) of the pump. The third term is the phenomenon called Photon Assisted Shockley-Read-Hall effect (PASRH) [30], corresponding to a two-step transition : the absorption of a pump photon into or from a sub-gap energy level induced by the material defects completed by a thermally activated transition. This PASRH is proportional to the pump intensity but the linear coefficient depends on a thermal absorption with many traps involved leading to complex predictions.

$$
\begin{aligned}
J_{\text {total }} & \propto\left(\beta\left(\omega_{p}, \omega_{s}\right) \int_{\mathrm{L}} I_{p} I_{s} d S+\beta\left(\omega_{p}, \omega_{p}\right) \int_{\mathrm{L}} I_{p}^{2} d S\right. \\
& \left.+\alpha\left(\omega_{p}\right) \int_{\mathrm{L}} I_{p} d S\right) P_{p}^{(\mathrm{i})} P_{s}^{(\mathrm{i})}
\end{aligned}
$$

A direct comparison of these three terms is difficult. Yet, some broad considerations can be made. First, a complete computation of $\beta$ [32] is illustrated in Fig. 2b and the two TPA processes (NDTPA and DTPA) are underlined. Since $\beta$ gets higher away from degeneracy (i.e. $\omega_{p} \gg \omega_{s}$ ), the NDTPA is favored.

Unfortunately, the PASRH also increases with the pump photon energy [29], thus a balance has to be found to increase the NDTPA while limiting the PASRH. In our configuration, $\beta_{\mathrm{NDTPA}}=87 \mathrm{~cm} \cdot \mathrm{GW}^{-1}$ is almost four times greater than $\beta_{\mathrm{DTPA}}=24 \mathrm{~cm} . \mathrm{GW}^{-1}$ while the pump energy represents $87 \%$ of the gap energy.

Since the structure should favor the NDTPA, the term $\int_{L} I_{p} I_{s}$ needs to be as high as possible. At the same time, the terms $\int_{L} I_{p}^{2}$ and $\int_{L} I_{p}$ must be as low as possible to limit the DTPA and PASRH. To illustrate this concept, the normalised pump intensity and signal intensity maps have been depicted on Fig. $3 \mathrm{a}$ and b. These maps illustrate the intensity distribution inside the structure and are thus related to the absorption processes that are possible at the various positions. Most importantly, the signal intensity map illustrates the location where NDTPA could happen and should be maximised. However the pump intensity map is more difficult to interpret. Indeed, while the pump intensity is needed to activate NDTPA, it also gives way to the competitive DTPA and PASRH processes. So we suggest to differentiate the "useful" pump intensity that contributes to NDTPA from the rest. This leads to the definition of the area $\mathcal{A}$ where $I_{S}$ is below a threshold that we arbitrary fix at $20 \%$ of its maximum (see Fig. $3 a$ ). We consider that the pump intensity located inside $\mathcal{A}$ cannot contribute to NDTPA and should be minimised. Conversely, the pump intensity located outside $\mathcal{A}$ can be interpreted as useful for NDTPA and has to be maximised (see Fig. 3c). The fraction of $I_{p}$ inside $\mathcal{A}$ which is detrimental to efficient photodetection through NDTPA is illustrated in Fig. 3d.

While only the gain G (see Eq. 3) was previously investigated in the literature to optimise the nanostructures due to its direct relation to NDTPA, we propose here to refine the optimisation process to tackle the issue of the pump distribution. A new figure of merit, called F, is built as the ratio of NDTPA over the avoidable DTPA and PASRH.

$$
F=\frac{\int_{\mathrm{L}} \widetilde{I}_{p} I_{s} d S}{\int_{\mathrm{L}} \tilde{I}_{p}^{2} d S \int_{\mathcal{A}} \tilde{I}_{p} d S} \text { with } \int_{\mathrm{L}} \widetilde{I}_{p} d S=1
$$

$\tilde{I}_{p}$ is the normalised pump intensity in the layer that enables the comparison between very different structures if one assumes the non-depletion of the pump power.

Furthermore a complementary optimisation is needed for infrared detection through NDTPA. Indeed, the optical beam is usually focused onto the detector. On the other hand the nanocavities resonances are known to spectral shift with the angle of incidence. As the Fig. 4 illustrates, the structure was optimised for a collimated pump with an incidence angle of $\theta_{p}^{i}=11.83^{\circ}$ and a collimated signal at normal incidence. The best configuration leads to a gain up to $G=120$ and a figure of merit up to $F=140$ $\mathrm{m}$. However, the gain and the figure of merit drop can be drastic with a modification of any one of the incident angles.

One can simulate the effect of a focused beam as the pondered mean of $F$ and $G$ over the cone of incidence angles - this is expected to greatly reduce both the gain and the figure of merit. Effectively, as the Fig. 4c and d illustrate both $G$ and $F$ drop for a focused pump beam (cone of half-angle $\varphi_{p}=2^{\circ}$ ). This observation brings the need for a refined optimisation procedure, which is driven by the aimed application of the photodetector. 


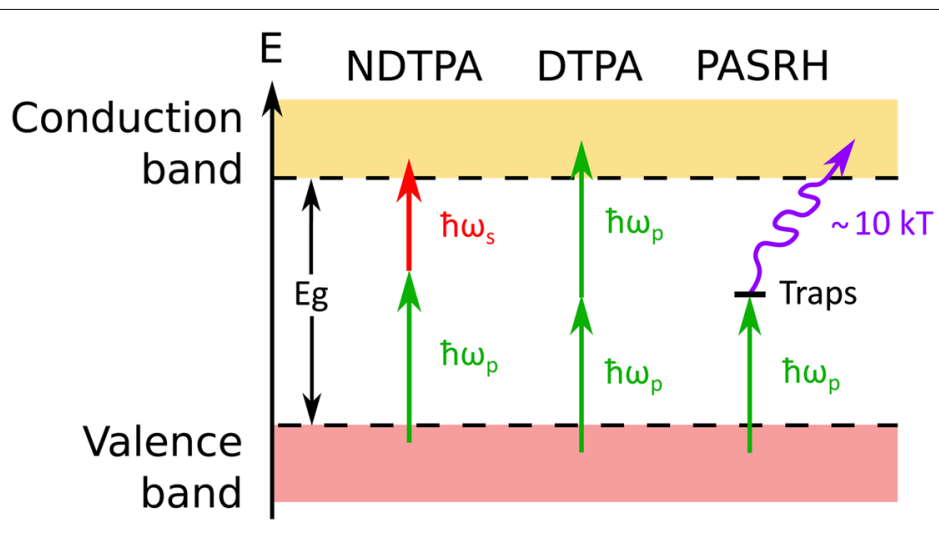

(a)

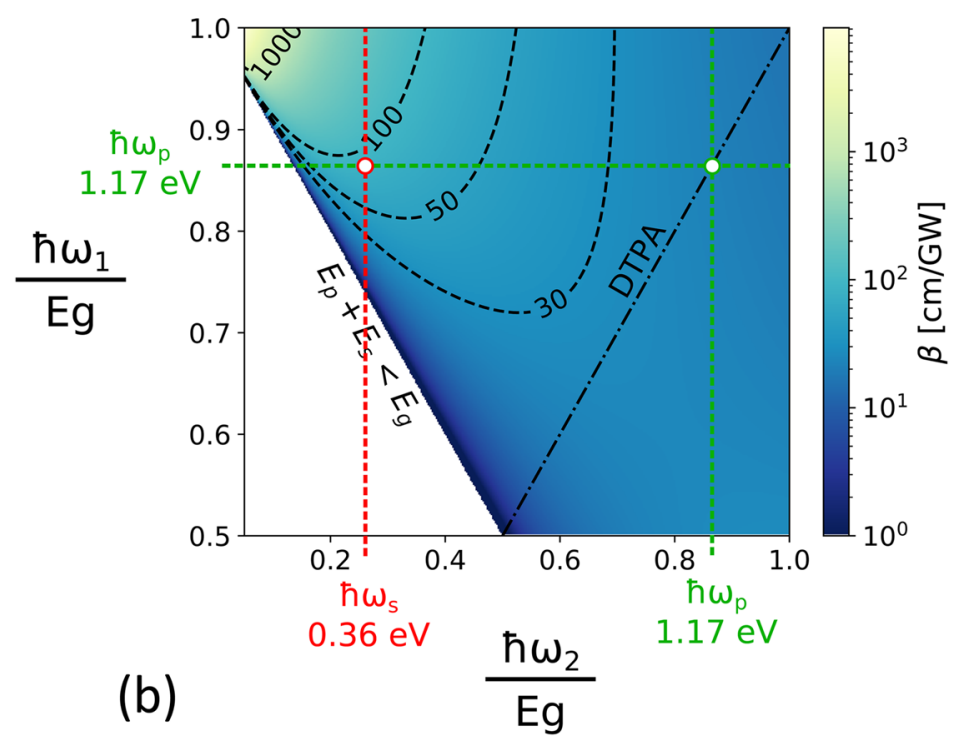

Fig. 2 (a). Illustration of the three competitive sub-bandgap absorption processes (NDTPA, DTPA and PASRH). (b). Evolution of the two-photon absorption coefficient of InP with regards to the signal and pump energy

(a)

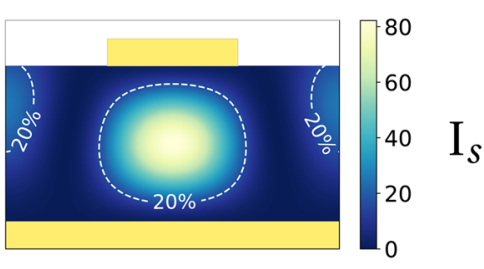

(c)

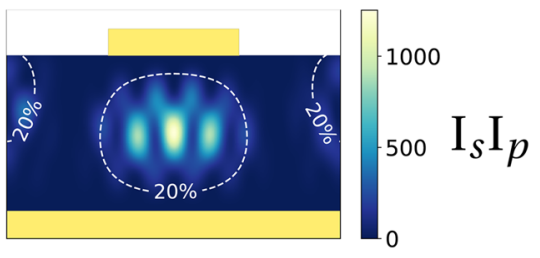

(b)

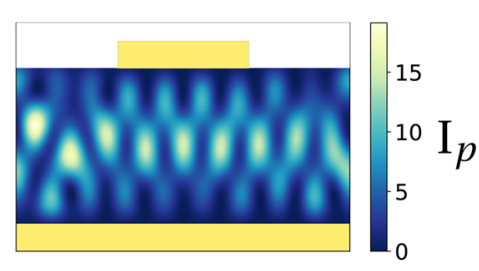

(d)

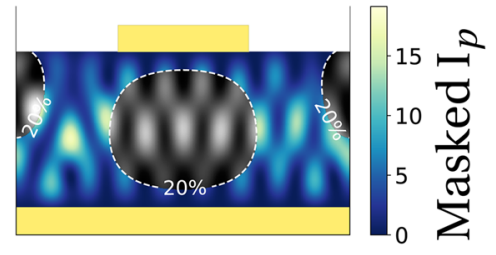

Fig. 3 (a). Signal intensity map. A white dotted isoline delimits the surface $\mathcal{A}$ where $I_{s}<20 \% I_{s}^{\max }$. (b). Pump intensity map (c). Intensity maps of the signal and pump colocalisation. NDTPA is mostly generated in this area. (d). Pump intensity map in $\mathcal{A}$. This pump intensity is detrimental since it mainly generates PASRH 


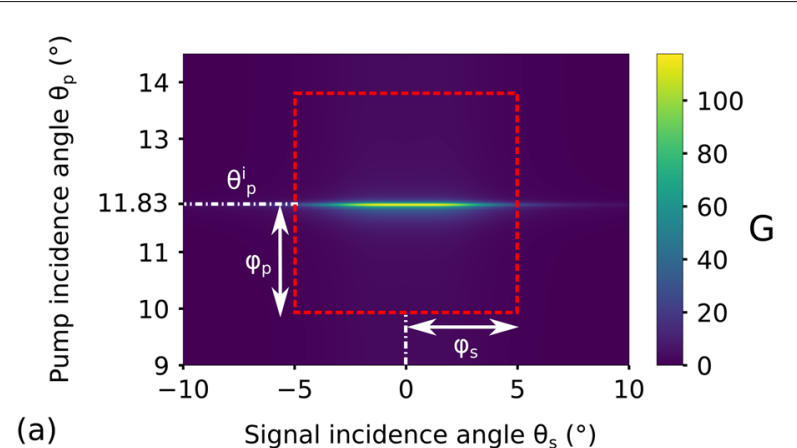

(a)

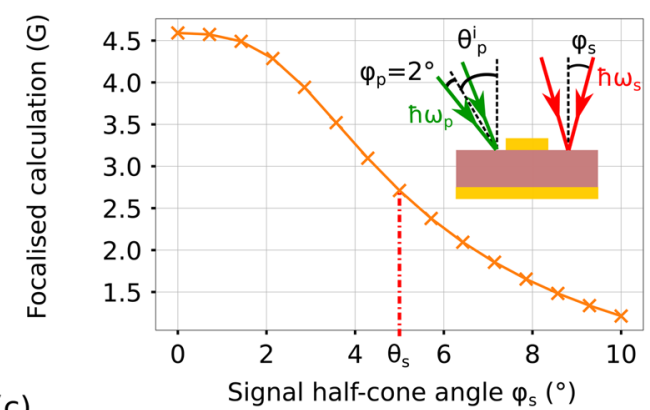

(c)
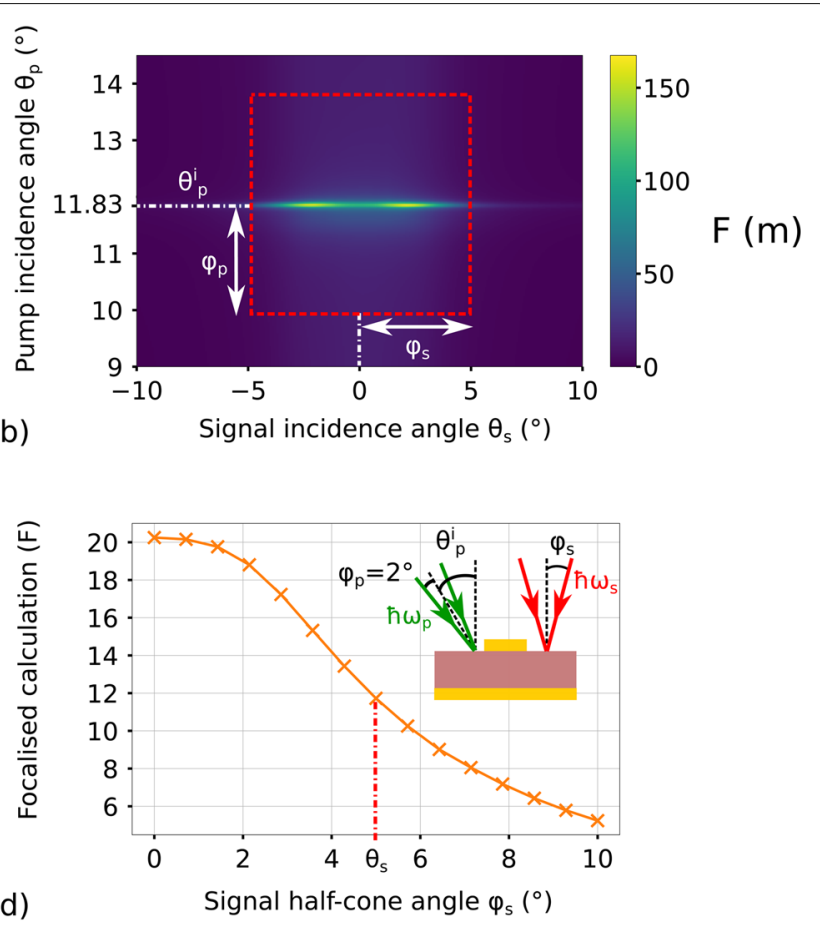

Fig. $4 \mathrm{G}$ (a) and $\mathrm{F}$ (b) for different incidence signal and pump angles. Both figures depict the best configurations for the detection. The calculation were performed for collimated beams. This structure needs a pump angle of $\theta_{p}^{i}=11.83^{\circ}$ to detect infrared light. The gaussian ponderated integration of $G$ and $F$ inside the rectangle leads to their focused value. Evolution of $G$ (c) and $F(d)$ values for a focused configuration with the signal half-cone angle $\varphi_{s}$ and a fixed pump half-cone angle of $\varphi_{p}=2.0^{\circ}$

We now propose to make use of our 3-step optimisation : maximisation of gain G (NDTPA enhancement), maximisation of figure of merit $F$ (sub-gap competition) and optimisation of angular tolerance (use of focused beams), in the configuration where a signal beam (around $3.39 \mu \mathrm{m})$ and a pump beam $(1.06 \mu \mathrm{m})$ are both focused on a photodiode. The methodology introduced here is still suitable to any infrared wavelength, according to the desired function. And thanks to the generality of this methodology, one can straightforwardly adapt the optimisation process to another specific application. To illustrate the flexibility of our optimisation methodology, two structures tackling different infrared photodetection scenarios are introduced.

The first structure is a nanostructured InP junction absorbing at $3.41 \mu \mathrm{m}$ (see Fig. 5a) for monochromatic LIDAR or heterodyne detection. We emphasize that, for this application, the spectral absorption bandwidth is not a relevant feature. Moreover, since heterodyne detection tends to operate with large pixels, the signal will not be strongly focused and the angular stability of $G$ or $F$ is not a crucial feature. Based on these principles, a guided mode is used for the signal concentration. This resonance seems well suited since, despite a low angular stability, it allows a field concentration that spreads over a large area of the junction. The signal distribution is mainly focused under the ends of the gold bar as well as into the lower half of the absorbing junction under the air slots. The pump resonance is based on a vertical Fabry-Perot which displays an interesting angular stability. The pump distribution is mainly focused as lobes under the air slots. Thus the signal and pump distributions overlap rather well under the air slots, leading to a great colocalisation. The fields and concentration maps are depicted in the supplementary materials. As illustrated on Fig. 5 and Table 1, the optimised structure presents, for a focused pump with a $2^{\circ}$ half-cone angle, a gain $G=35$ and a figure of merit $F=108 \mathrm{~m}$ that are respectively 7 and 5 times higher than those of the literature. The angular stability of $\mathrm{G}$ and $\mathrm{F}$ is about $3^{\circ}$ which is coherent with the application. In addition, it is noteworthy that, contrary to the structure from literature, both pump and signal are working at normal incidence which highly simplifies the use of such photodetectors.

The second structure is a nanostructured gallium arsenide (GaAs) junction with an extended absorption band in the MWIR (3-4 $\mu \mathrm{m})$ for imaging applications. In this regard, the device requires both a wide spectral absorption band and an angular stability at least up to $10^{\circ}$. To this end a coupled nano-Fabry Perot (nFPc) [33] 


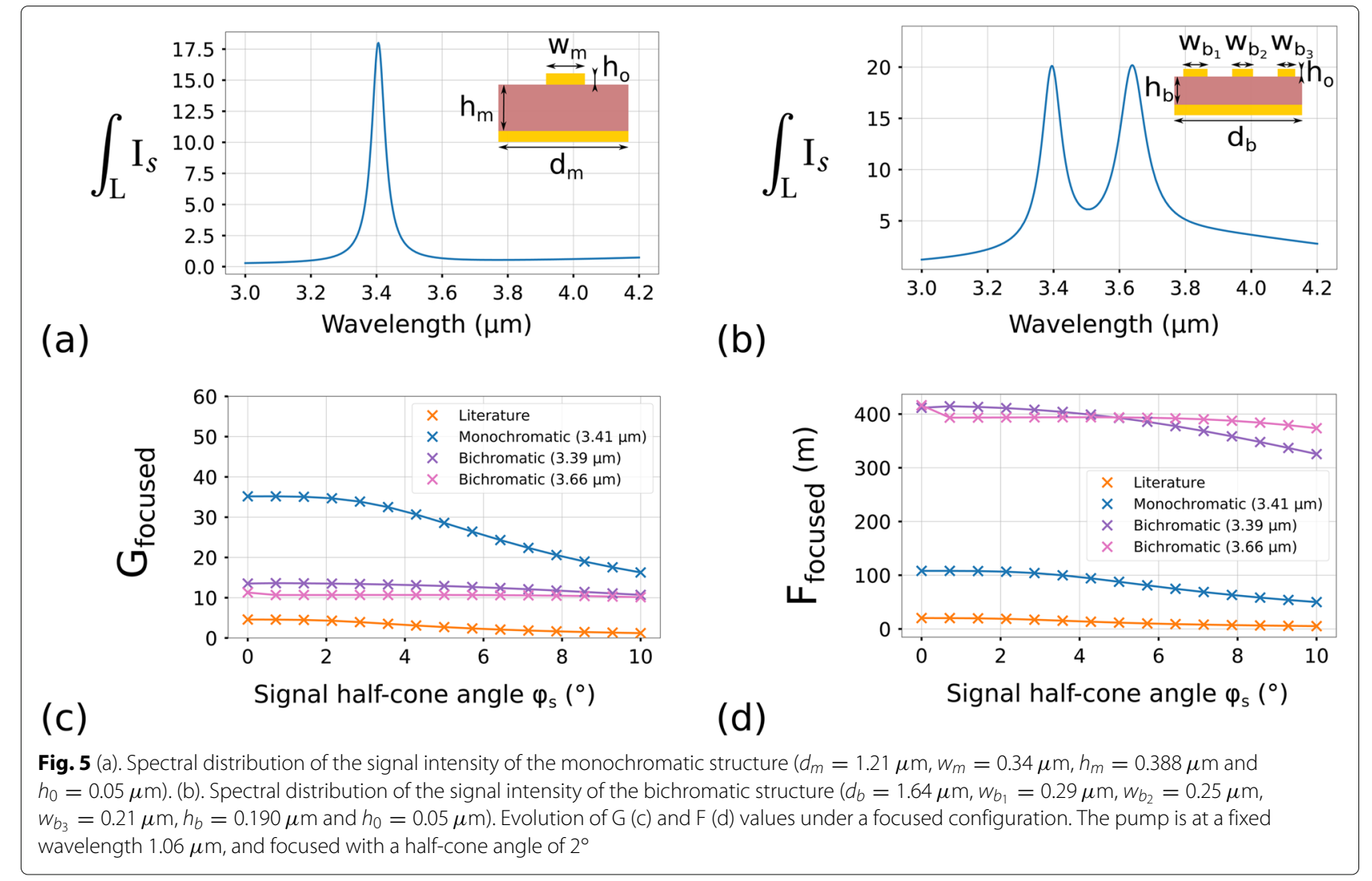

resonator is used for the signal concentration. The nFPc enables to design several resonances with similar field distribution and high angular tolerance. The signal distribution is mainly concentrated under the ends of two side-by-side gold bars until it extends under the air gap between them in the lower half of the absorbing junction. The pump resonance is based on a guided mode resonance. Thus the pump distribution is distributed as a lobe along the absorbing junction. The signal and pump distributions overlap very well considering the two detectable signal wavelengths. Few signal-free pump regions are available, which will lead to a very good figure of merit F. The fields and concentration maps are depicted in the supplementary materials. As illustrated on Fig. 5 and Table 1 , the angular mean values of $\mathrm{G}(3.39 \mu \mathrm{m})=$ 10.6 and $\mathrm{G}(3.66 \mu \mathrm{m})=12.6$ are about 4 times higher than the angular mean value of the literature $G=2.88$ [22]. As expected, the increased spectral bandwidth and the angular stability come at the cost of 3 times lower gain at normal incidence as compared to the one of the monochromatic structure. With regards of the figure of merit, the introduction of a multispectral nanostructure imposes to redefine the detrimental pump intensity. Indeed, the pump intensity that is colocalised with either signal intensity can enable NDTPA. Thus the area $\mathcal{A}$ has to be defined with regards to each signal resonance such as : $\mathcal{A}=\mathcal{A}_{3.39 \mu \mathrm{m}} \cup \mathcal{A}_{3.66 \mu \mathrm{m}}$. Finally, the figure of merit $\mathrm{F}$ is almost constant with regard to the signal half-cone angle which is relevant with the expectations.

Thus two structures with distinct objectives have been depicted, based on an optimisation process through the

Table 1 Gain $G$ and figure of merit $F$ values depicted for all the structures

\begin{tabular}{|c|c|c|c|c|c|c|}
\hline Structure & Signal resonator & Pump resonator & $\mathrm{G}\left(0^{\circ}\right)$ & $\mathrm{G}\left(10^{\circ}\right)$ & $\mathrm{F}\left(0^{\circ}\right)[\mathrm{m}]$ & $\mathrm{F}\left(10^{\circ}\right)[\mathrm{m}]$ \\
\hline Literature [22] & Diffraction order & GMR & 4.60 & 1.20 & 20.2 & 5.24 \\
\hline Monochromatic & Guided mode & Vertical Fabry-Perot & 35.2 & 16.3 & 108 & 50.0 \\
\hline Bichromatic (3.39 $\mu \mathrm{m})$ & $\mathrm{nFPc}$ & GMR & 13.5 & 10.7 & 411 & 325 \\
\hline Bichromatic $(3.66 \mu \mathrm{m})$ & $n F P c$ & GMR & 11.3 & 10.1 & 416 & 373 \\
\hline
\end{tabular}

The computation is performed for a focused pump with a half-cone angle of $2^{\circ}$. The signal is either collimated $\left(0^{\circ}\right)$ or focused with a half-cone angle of $10^{\circ}$ 
figure of merit design. Consequently the resonator choice is made to meet the device requirements. Many configurations may be possible for the desired application but the figure of merit sets up points of comparison for every case. Therefore this methodology is flexible and suitable for various devices and tunable for various spectral configurations.

\section{Conclusion}

In conclusion, a methodology to optimise two-photon photodetection in nanostructured detectors for low power infrared sensors was described. Based on the NDTPA equations and its competition with two other sub-bandgap absorption processes, we suggested a 3-step optimisation procedure. First comes the structure gain, which quantifies NDTPA through the colocatisation of the pump and the signal intensities. Second the ratio of NDTPA over the avoidable DTPA and PASRH that can be interpreted as a signal to noise optimisation. And third comes the structure angular tolerance, which is required for most applications where the optical beams are focused. As a result, the optimisation methodology was demonstrated for two structures aiming at distinct scenarios. This optimisation methodology can easily be adapted to various wavelengthes, various absorbing junctions and various applications; it paves the way to new efficient designs.

\section{Supplementary Information}

The online version contains supplementary material available at

https://doi.org/10.1186/s41476-021-00167-6.

Additional file 1: Supplementary materials.

Acknowledgements

This work was supported by a DGA-AID scholarship.

\section{Authors' contributions}

$\mathrm{BF}$ initiated and coordinated the project. MD is the author of this manuscript. $\mathrm{MD}$ and BF led the theoretical work. JJ and RH largely participated in structuring and editing the project through numerous fruitful discussions. BF, $\mathrm{JJ}$ and $\mathrm{RH}$ also helped extensively with the manuscript corrections. All authors agreed on their own contributions. And all authors read and approved the final manuscript.

\section{Authors' information}

1. ONERA,6 Chemin de la Vauve aux Granges, 91123 Palaiseau Cedex, France. 2. École polytechnique (Département de Physique), Route de Saclay, 91128 Palaiseau, France.

\section{Funding}

This work is supported by a DGA-AID (Direction Générale de l'Armement Agence innovation Défense) and ONERA (Office National d'études et de Recherches Aérospatiales) funding

Availability of data and materials Not applicable.

\section{Declarations}

\section{Competing interests}

The authors declare that they have no competing interests.

\section{Author details}

${ }^{1}$ DOTA, ONERA, Université Paris-Saclay, 91123 Palaiseau, France. ${ }^{2}$ École Polytechnique, Département de Physique, 91128 Palaiseau, France.

Received: 7 May 2021 Accepted: 7 July 2021

Published online: 18 September 2021

References

1. Rogalski, A.: HgCdTe photodetectors. Woodhead Publishing Series in Electronic and Optical Materials, 235-335 (2020). https://doi.org/10.1016/ B978-0-08-102709-7.00007-3. https://www.sciencedirect.com/science/ article/pii/B9780081027097000073. Accessed 19 Apr 2021

2. Klipstein, P., Aronov, D., Ezra, M. b., Barkai, I., Berkowicz, E., Brumer, M., Fraenkel, R., Glozman, A., Grossman, S., Jacobsohn, E., Klin, O., Lukomsky, I., Shkedy, L., Shtrichman, I., Snapi, N., Yassen, M., Weiss, E.: Recent progress in InSb based quantum detectors in Israel. Infrared Phys. Technol. 59, 172-181 (2013). https://doi.org/10.1016/j.infrared.2012.12.035. Accessed 20 Apr 2021

3. Rogalski, A.: Quantum well photoconductors in infrared detector technology. J. Appl. Phys. 93, 4355-4391 (2003). https://doi.org/10.1063/ 1.1558224

4. Choi, K. K., Jhabvala, M. D., Sun, J., Jhabvala, C. A., Waczynski, A., Olver, K.: Resonator-quantum well infrared photodetectors. Appl. Phys. Lett. 103(20), 201113 (2013). https://doi.org/10.1063/1.4831797. Publisher: American Institute of Physics. Accessed 20 Apr 2021

5. Ivanov, R., Smuk, S., Hellström, S., Evans, D., Höglund, L., Costard, E.: LWIR QWIPs at IRnova for next generation polarimetric imaging. Infrared Phys. Technol. 95 (2018). https://doi.org/10.1016/j.infrared.2018.10.017

6. Rogalski, A., Kopytko, M., Martyniuk, P.: InAs/GaSb type-Il superlattice infrared detectors: three decades of development, p. 1017715, (2017). https://doi.org/10.1117/12.2272817

7. Rogalski, A., Martyniuk, P., Kopytko, M.: Type-II superlattice photodetectors versus HgCdTe photodiodes. Prog. Quantum Electron. 68, 100228 (2019). https://doi.org/10.1016/j.pquantelec.2019.100228. Accessed 19 Apr 2021

8. Rogalski, A., Martyniuk, P., Kopytko, M., Hu, W.: Trends in Performance Limits of the HOT Infrared Photodetectors. Appl. Sci. 11(2), 501 (2021). https://doi.org/10.3390/app11020501. Accessed 08 Mar 2021

9. Langrock, C., Diamanti, E., Roussev, R. V., Yamamoto, Y., Fejer, M. M., Takesue, H.: Highly efficient single-photon detection at communication wavelengths by use of upconversion in reverse-proton-exchanged periodically poled $\mathrm{LiNbO}_{3}$ waveguides. Opt. Lett. 30(13), 1725-1727 (2005). https://doi.org/10.1364/OL.30.001725. Publisher: Optical Society of America. Accessed 22 Mar 2021

10. Boitier, F., Godard, A., Rosencher, E., Fabre, C.: Measuring photon bunching at ultrashort timescale by two-photon absorption in semiconductors. Nat Phys. 5(4), 267-270 (2009). https://doi.org/10.1038/nphys1218. Number: 4 Publisher: Nature Publishing Group. Accessed 14 Jan 2021

11. Fishman, D. A., Cirloganu, C. M., Webster, S., Padilha, L. A., Monroe, M., Hagan, D. J., Van Stryland, E. W.: Sensitive mid-infrared detection in wide-bandgap semiconductors using extreme non-degenerate two-photon absorption. Nat. Photon. 5(9), 561-565 (2011). https://doi. org/10.1038/nphoton.2011.168. Number: 9 Publisher: Nature Publishing Group. Accessed 19 Mar 2021

12. Portier, B., Vest, B., Pardo, F., Péré-Laperne, N., Steveler, E., Jaeck, J., Dupuis, C., Bardou, N., Lemaître, A., Rosencher, E., Haïdar, R., Pelouard, J.-L.: Resonant metallic nanostructure for enhanced two-photon absorption in a thin GaAs p-i-n diode. Appl. Phys. Lett. 105(1), 011108 (2014). https:// doi.org/10.1063/1.4887375. Publisher: American Institute of Physics. Accessed 14 Jan 2021

13. Hutchings, D. C., Stryland, E. W. V.: Nondegenerate two-photon absorption in zinc blende semiconductors. JOSA B. 9(11), 2065-2074 (1992). https://doi.org/10.1364/JOSAB.9.002065. Publisher: Optical Society of America. Accessed 10 Mar 2021

14. Stryland, E. W. V., Woodall, M. A., Vanherzeele, H., Soileau, M. J.: Energy band-gap dependence of two-photon absorption. Opt. Lett. 10(10), 490-492 (1985). https://doi.org/10.1364/OL.10.000490. Publisher: Optical Society of America. Accessed 09 Mar 2021 
15. Nga Chen, Y., Todorov, Y., Askenazi, B., Vasanelli, A., Biasiol, G., Colombelli, R., Sirtori, C.: Antenna-coupled microcavities for enhanced infrared photo-detection. Appl. Phys. Lett. 104(3), 031113 (2014). https://doi.org/ 10.1063/1.4862750. Accessed 09 Mar 2021

16. Koechlin, C., Bouchon, P., Pardo, F., Pelouard, J.-L., Haïdar, R.: Analytical description of subwavelength plasmonic MIM resonators and of their combination. Opt. Express. 21(6), 7025 (2013). https://doi.org/10.1364/OE. 21.007025. Accessed 04 Jan 2021

17. Verdun, M., Portier, B., Jaworowicz, K., Jaeck, J., Lelarge, F., Guilet, S., Dupuis, C., Haïdar, R., Pardo, F., Pelouard, J.-L.: Guided-mode resonator for thin InGaAs P-i-N short-wave infrared photo-diode. Appl. Phys. Lett. 108(5), 053501 (2016). https://doi.org/10.1063/1.4941297. Accessed 09 Mar 2021

18. Fabas, A., El Ouazzani, H., Hugonin, J.-P., Haïdar, R., Greffet, J.-J., Bouchon, P.: Helmholtz-like Nanoresonators Applied to Surface Enhanced Infrared Absorption. In: Metamaterials 2019, Rome, (2019). https://hal.archivesouvertes.fr/hal-02409551. Accessed 29 Apr 2021

19. Palaferri, D., Todorov, Y., Bigioli, A., Mottaghizadeh, A., Gacemi, D., Calabrese, A., Vasanelli, A., Li, L., Davies, A. G., Linfield, E. H., Kapsalidis, F., Beck, M., Faist, J., Sirtori, C.: Room-temperature nine- $\mu$ m-wavelength photodetectors and $\mathrm{GHz}$-frequency heterodyne receivers. Nature. 556(7699), 85-88 (2018). https://doi.org/10.1038/nature25790. Accessed 09 Mar 2021

20. Miyazaki, H. T., Mano, T., Kasaya, T., Osato, H., Watanabe, K., Sugimoto, Y., Kawazu, T., Arai, Y., Shigetou, A., Ochiai, T., Jimba, Y., Miyazaki, H.: Synchronously wired infrared antennas for resonant single-quantum-well photodetection up to room temperature. 10 (2020). https://doi.org/10. 1038/s41467-020-14426-6

21. Hakl, M., Lin, Q., Lepillet, S., Billet, M., Lampin, J.-F., Pirotta, S., Colombelli, R. Wan, W., Cao, J. C., Li, H., Peytavit, E., Barbieri, S.: Ultrafast Quantum-Well Photodetectors Operating at 10 um with a Flat Frequency Response up to $70 \mathrm{GHz}$ at Room Temperature. ACS Photonics. 8(2), 464-471 (2021). https://doi.org/10.1021/acsphotonics.0c01299. Publisher: American Chemical Society. Accessed 09 Mar 2021

22. Fix, B., Jaeck, J., Vest, B., Verdun, M., Beaudoin, G., Sagnes, I., Pelouard, J.-L., Haïdar, R.: Nanostructured diode for infrared photodetection through nondegenerate two-photon absorption. Appl. Phys. Lett. 111(4), 041102 (2017). https://doi.org/10.1063/1.4996369. Accessed 04 Feb 2021

23. Xu, G., Ren, X., Miao, Q., Yan, M., Pan, H., Chen, X., Wu, G., Wu, E.: Sensitive Infrared Photon Counting Detection by Nondegenerate Two-Photon Absorption in Si APD. IEEE Photon. Technol. Lett. 31(24), 1944-1947 (2019). https://doi.org/10.1109/LPT.2019.2950542

24. Fang, J., Wang, Y., Yan, M., Wu, E., Huang, K., Zeng, H.: Highly Sensitive Detection of Infrared Photons by Nondegenerate Two-Photon Absorption Under Midinfrared Pumping. Phys. Rev. Appl. 14(6), 064035 (2020). https:// doi.org/10.1103/PhysRevApplied.14.064035. Accessed 09 Mar 2021

25. Bouchon, P., Pardo, F., Haïdar, R., Pelouard, J.-L.: Fast modal method for subwavelength gratings based on B-spline formulation. J. Opt. Soc. Am. A. 27(4), 696 (2010). https://doi.org/10.1364/JOSAA.27.000696. Accessed 03 Mar 2021

26. Levinshtein, M., Rumyantsev, S., Shur, M.: Handbook Series on Semiconductor Parameters: Volume 1: Si, Ge, C (Diamond), GaAs, GaP, GaSb, InAs, InP, InSb. vol. 1. World Scientific (1996). https://doi.org/10. 1142/2046-vol1. https://www.worldscientific.com/worldscibooks/10. 1142/2046-vol1. Accessed 12 Mar 2021

27. Vavilov, V. S.: Handbook on the physical properties of Ge, Si, GaAs and InP by A Dargys and J Kundrotas. Physics-Uspekhi. 39(7), 757-757 (1996). https://doi.org/10.1070/PU1996v039n07ABEH001526. Accessed 12 Mar 2021

28. Nayak, S. K., Sahu, T., Mohanty, S. P.: Third-order nonlinear optical susceptibilities of group IV and III-V compound semiconductors. Phys. B. Condens. Matter. 191(3), 334-340 (1993). https://doi.org/10.1016/09214526(93)90093-L. Accessed 20 Apr 2021

29. Vest, B., Fix, B., Jaeck, J., Haïdar, R.: Competition between sub-bandgap linear detection and degenerate two-photon absorption in gallium arsenide photodiodes. J. Eur. Opt. Society-Rapid Publ. 12(1), 26 (2016). https://doi.org/10.1186/s41476-016-0022-8. Accessed 04 Feb 2021

30. Vest, B., Lucas, E., Jaeck, J., Haïdar, R., Rosencher, E.: Silicon sub-bandgap photon linear detection in two-photon experiments: A photo-assisted Shockley-Read-Hall mechanism. Appl. Phys. Lett. 102(3), 031105 (2013). https://doi.org/10.1063/1.4788705. Publisher: American Institute of Physics. Accessed 14 Jan 2021
31. Piccardo, M., Rubin, N. A., Meadowcroft, L., Chevalier, P., Yuan, H., Kimchi, J., Capasso, F.: Mid-infrared two-photon absorption in an extended-wavelength InGaAs photodetector. Appl. Phys. Lett. 112(4), 041106 (2018). https://doi.org/10.1063/1.5018619. Accessed 09 Mar 2021

32. Cirloganu, C. M., Padilha, L. A., Fishman, D. A., Webster, S., Hagan, D. J., Stryland, E. W. V.: Extremely nondegenerate two-photon absorption in direct-gap semiconductors [Invited]. Opt. Express. 19(23), 22951-22960 (2011). https://doi.org/10.1364/OE.19.022951. Publisher: Optical Society of America. Accessed 10 Mar 2021

33. Fix, B., Jaeck, J., Bouchon, P., Héron, S., Vest, B., Haïdar, R.: High-quality-factor double Fabry-Perot plasmonic nanoresonator. Opt. Lett. 42(24), 5062-5065 (2017). https://doi.org/10.1364/OL.42.005062. Publisher: Optical Society of America. Accessed 22 Mar 2021

\section{Publisher's Note}

Springer Nature remains neutral with regard to jurisdictional claims in published maps and institutional affiliations.

\section{Submit your manuscript to a SpringerOpen ${ }^{\circ}$ journal and benefit from:}

- Convenient online submission

- Rigorous peer review

- Open access: articles freely available online

- High visibility within the field

- Retaining the copyright to your article

Submit your next manuscript at $\gg$ springeropen.com 